Powell, J. F. \& Hunter, J. R. (1955). J. gen. Microbiol. 13, 59-67.

\title{
Spore Germination in the Genus Bacillus: the Modification of Germination Requirements as a Result of Preheating
}

\author{
By JOAN F. POWELL AND J. R. HUNTER \\ Microbiological Research Department (Ministry of Supply), Porton, Wiltshire
}

SUMMARY: Spore suspensions of Bacillus megaterium germinated spontaneously after heat treatment. The extent of germination depended on the degree of close packing of the suspension, the temperature and duration of heating, and on the presence of water. Spontaneous germination was not inhibited by heating with cyanide, azide, 2:4-dinitrophenol or sodium iodoacetate, but was significantly inhibited by sodium fluoride.

Freshly harvested spores of a laboratory strain of Bacillus cereus required either inosine or a mixture of alanine + tyrosine + adenosine for optimal germination. After prolonged storage or a short heat treatment, adenosine alone stimulated rapid and complete germination. Heat activation did not occur in the absence of water. It was inhibited to some extent by sodium fluoride. Similar results were obtained with $B$. cereus, NCTC 8035.

After preheating, spores of a virulent and an avirulent strain of Bacillus anthracis germinated more rapidly and completely in a mixture of adenosine + tyrosine + alanine, but not in adenosine alone. Inosine stimulated rapid and complete germination of heated suspensions, and was consistently more effective than adenosine. Adenosine deaminase activity has been found in extracts from resting spores of the laboratory $B$. cereus and the avirulent strain of $B$. anthracis but not in $B$. megaterium.

As in previous studies, the criterion of germination was loss of heat resistance accompanied by a change in staining properties, decrease of refractive index and decrease in dry weight (Powell \& Strange, 1953). A laboratory strain of Bacillus megaterium which required glucose specifically for spore germination was described by Powell (1951). Some freshly harvested spore suspensions of this organism germinated very poorly in glucose solution but were activated by heating at $60^{\circ}$ for $30 \mathrm{~min}$. Other suspensions, grown on the same medium germinated completely in glucose solution without heat activation. It was later accidentally discovered that when thick spore suspensions $\left(2 \times 10^{10}\right.$ spores $/ \mathrm{ml}$.) were heated at $60^{\circ}$ for 1 to $2 \mathrm{hr}$., then centrifuged and resuspended in water, spontaneous and complete germination occurred without loss of viability. This behaviour provided a means of obtaining concentrated germination exudate (Powell \& Strange, 1953). In suspensions thicker than $1 \times 10^{10}$ spores/ml. the amount of calcium dipicolinate excreted (Powell, 1953) was actually greater than the saturation concentration, and the substance appeared as a heavy white granular deposit. We have now studied this heat-activated germination in some detail. The modification of germination requirements as a result of preheating has also been studied in two strains of $B$. cereus and in a virulent and an avirulent strain of $\boldsymbol{B}$. anthracis. Freshly harvested, unheated spore suspensions of these organisms germinated in a mixture of L-alanine + tyrosine + adenosine (Hills, 1949). 
Several examples of the stimulatory effect of preheating on spore germination in bacteria and fungi have already been described (Goddard, 1939; Hull, 1939; Curran \& Evans, 1945; Powell, 1950). The present report describes further examples of this effect, and some attempts to define the stimulation in chemical terms.

\section{ORGANISMS AND METHODS}

Washed suspensions of spores of Bacillus megaterium were prepared as described previously (Powell, 1951). Spores of the laboratory strain of $B$. cereus, of $B$. cereus NCTC 8035 and of an avirulent strain of $B$. anthracis were obtained after $48 \mathrm{hr}$. growth at $37^{\circ}$ on potato-extract agar (Robinow, 1951 ) enriched with $1 / 10 \mathrm{vol}$. of casein hydrolysate-yeast extract medium CCY (Gladstone \& Fildes, 1940). Spores of the virulent strain of B. anthracis were obtained by $48 \mathrm{hr}$. growth on CCY agar. Spore suspensions were washed thoroughly with water at $3^{\circ}$ until they were free from cell debris, i.e. at least five times, and stored at $3^{\circ}$. It was generally possible to obtain suspensions containing less than 5\% densely staining, non-viable forms (Powell, 1951) and such suspensions, with the exception of those from the laboratory strain of $B$. cereus, were stable at $3^{\circ}$. Spore suspensions of the laboratory strain of $B$. cereus gradually deteriorated owing to spontaneous germination followed by disintegration and were discarded when the proportion of densely staining forms became greater than $5 \%$.

All the germination tests were made at $37^{\circ}$, unless otherwise stated, in the presence of $40 \mathrm{~mm}$-phosphate $(\mathrm{pH} 7 \cdot 3)$ at concentrations of 0.5 to $1.0 \times 10^{9}$ spores $/ \mathrm{ml}$. The percentage germination was assessed by the staining technique and the speed of germination by repeated measurements of light scattering (Powell, 1950).

For the detection of adenosine deaminase activity, suspensions containing $2 \times 10^{10}$ spores $/ \mathrm{ml}$. in $50 \mathrm{~mm}$-phosphate buffer $(\mathrm{pH} 7 \cdot 3)$ were first broken down mechanically at $3^{\circ}$ in a tissue disintegrator (Mickle, 1948) with Ballotini beads (size 12). The enzyme was present in the clear extract obtained by high-speed centrifuging. This extract was incubated for $30 \mathrm{~min}$. with $1 \mathrm{~mm}$-adenosine, and the ultraviolet absorbing constituents were separated using the paper ionophoresis technique of Wade \& Morgan (1954). After $2 \mathrm{hr}$. of ionophoresis the paper was dried and examined in ultraviolet light. Adenosine was separated from the slower-moving inosine by approximately $3 \mathrm{~cm}$. on the paper. Disintegrated spore extracts incubated without adenosine were also examined as controls.

\section{RESULTS}

Spontaneous germination of Bacillus megaterium spores after heat activation A suspension of Bacillus megaterium spores containing $\mathbf{1 . 5} \times 10^{10}$ spores $/ \mathrm{ml}$. was heated in $2 \mathrm{ml}$. batches for $15,30,60,90$ and $120 \mathrm{~min}$. at temperatures between $50^{\circ}$ and $78^{\circ}$. After centrifuging for $30 \mathrm{~min}$. at room temperature $\left(20^{\circ}\right)$ the spores were resuspended in $10 \mathrm{ml}$. of water, allowed to remain at room temperature for $1 \mathrm{hr}$. and the percentage germination determined by the 
staining method. When this was plotted against duration of heat treatment a sigmoid curve was obtained for each temperature (Fig. 1). Plotting temperature of activation against the period of heating required to give $50 \%$ germination, a smooth curve (Fig. 2) was obtained which suggested that very long heating periods would be necessary for activation at temperatures lower than $\mathbf{5 5 ^ { \circ }}$, and that there might be a critical temperature of activation. This appeared to be the case: almost complete germination occurred after $18 \mathrm{hr}$. heating at $45^{\circ}$ and practically no germination after $18 \mathrm{hr}$. at $44^{\circ}$. Different

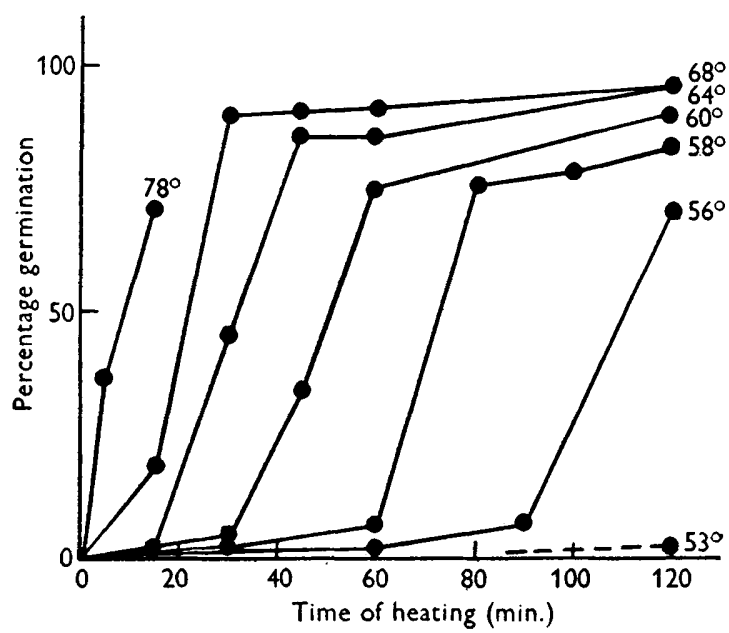

Fig. 1

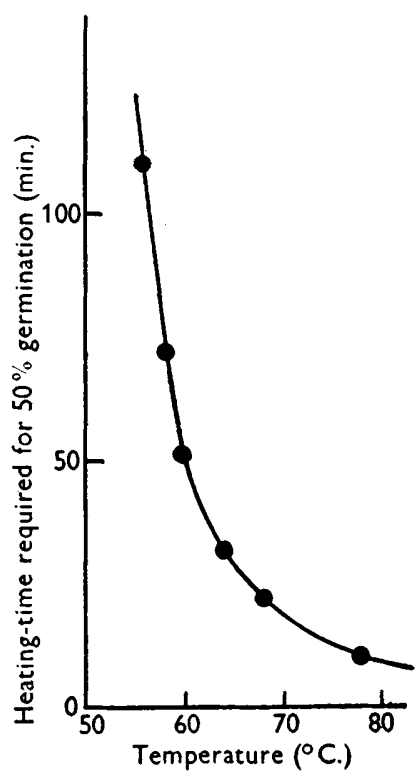

Fig. 2

Fig. 1. Heat-activated spontaneous germination of $B$. megaterium spores.

Fig. 2. Heat-activated spontaneous germination of $B$. megaterium spores. Data from Fig. 1.

spore crops showed similar but not identical behaviour to that just described. We have the impression that crops grown from cultures which showed strong melanin pigmentation, i.e. a high tyrosinase activity, were considerably more responsive to heat activation. Such crops also had a greater tendency to spontaneous germination during harvesting.

During these experiments it was noticed that a white granular deposit of calcium dipicolinate was present in the centrifuged mass of spores before resuspension. It thus seemed that germination proceeded while the spores were packed down, and not during resuspension as was first thought. It was then shown that resuspension was not an important factor in promoting spontaneous germination but that close packing after heat treatment was. Thus a spore suspension heated at $56^{\circ}$ for $2 \mathrm{hr}$. gave only $25 \%$ germination when allowed to stand without centrifuging, and almost complete germination when centrifuged and then simply shaken up. It seemed possible that a com- 
paratively small proportion of spores germinating in the packed cell mass was producing a very concentrated germination exudate which itself had a stimulatory effect on the germination of the remainder. This appeared to be the case. A suspension containing $1.5 \times 10^{10}$ spores $/ \mathrm{ml}$. was heated at $56^{\circ}$ for $2 \mathrm{hr}$, then transferred in $2.5 \mathrm{ml}$. lots to tubes containing 20 or $10 \mathrm{mg}$. of freeze-dried germination exudate. Germination in these tubes after $1 \mathrm{hr}$. standing was 61 and $41 \%$ respectively. A control tube of spores alone without addition of germination exudate showed only $18 \%$ germination. When the tubes were kept at $37^{\circ}$ for $1 \mathrm{hr}$. after heat treatment and addition of exudate, the corresponding germination figures were 90 and $82 \%$, with a control of $36 \%$. The constituent of the germination exudate responsible for this stimulation has not yet been identified. The isolated exudate fractions, i.e. calcium dipicolinate and the non-dialysable peptide (Strange \& Powell, 1954) were tested and found inactive.

As indicated above, a greater degree of germination was obtained by maintaining heat-activated suspensions at $37^{\circ}$ than at room temperature $\left(20^{\circ}\right)$. On the other hand, spontaneous germination was completely suppressed by cooling the heated suspension rapidly in ice. No germination occurred during heat treatment, and a suspension could be heated and ice-cooled alternately many times without spontaneous germination occurring. When, finally, the heated suspension was centrifuged and maintained at $37^{\circ}$ it germinated completely. It therefore appeared that the heat-sensitive system was activated by high temperatures but could only function between certain limits of temperature.

The heat-activation process was dependent on the presence of available water. Freeze-dried spores were heated at $60^{\circ}$ for $2 \mathrm{hr}$. in a stoppered tube containing $\mathrm{P}_{2} \mathrm{O}_{5}$, then suspended in water at $37^{\circ}$ and centrifuged; no spontaneous germination occurred. Similarly, when freeze-dried spores were heated in glycerol, ethanol, ethylene glycol or a saturated solution of KI, then diluted with water, centrifuged and resuspended, no germination occurred. Control tubes containing freeze-dried spores and water germinated completely after heating and centrifuging.

Spore suspensions were also heated for $2 \mathrm{hr}$. at $60^{\circ}$ in $1 \mathrm{~mm}$-potassium cyanide, 5 mm-sodium azide, 5 mm-2:4-dinitrophenol or 20 mm-sodium iodoacetate, then centrifuged and resuspended in water; no inhibition of heatactivated germination occurred. On heating in $100 \mathrm{~mm}$-sodium fluoride, germination was reduced from $82 \%$ in the control tube to $20 \%$. In a similar experiment with a different crop of spores, $100 \mathrm{~mm}$-fluoride reduced germination from 80 to $52 \%$.

\section{Germination of spores of Bacillus cereus and}

Bacillus anthracis

The rate of germination of spores of the laboratory strain of Bacillus cereus in tryptic digest broth (TMB), in adenosine + tyrosine + alanine all at $1 \mathrm{~mm}$ (AdTAl) and in 1 mm-adenosine was first compared, using the nephelometer 
method. Germination was generally complete, i.e. $>95 \%$ within 5 min. in TMB and in the AdTAl mixture. Different spore crops showed differing behaviour with adenosine. Usually, germination in adenosine was complete in $15 \mathrm{~min}$., but in some cases, only $50 \%$ germination occurred in $1 \mathrm{hr}$. and this was not increased during a further incubation for $1 \mathrm{hr}$. The effect of preheating aqueous spore suspensions at $60^{\circ}$ for $1 \mathrm{hr}$. was, in all cases $(a)$ to reduce the time required for complete germination in TMB and AdTAl to 2-3 min., and

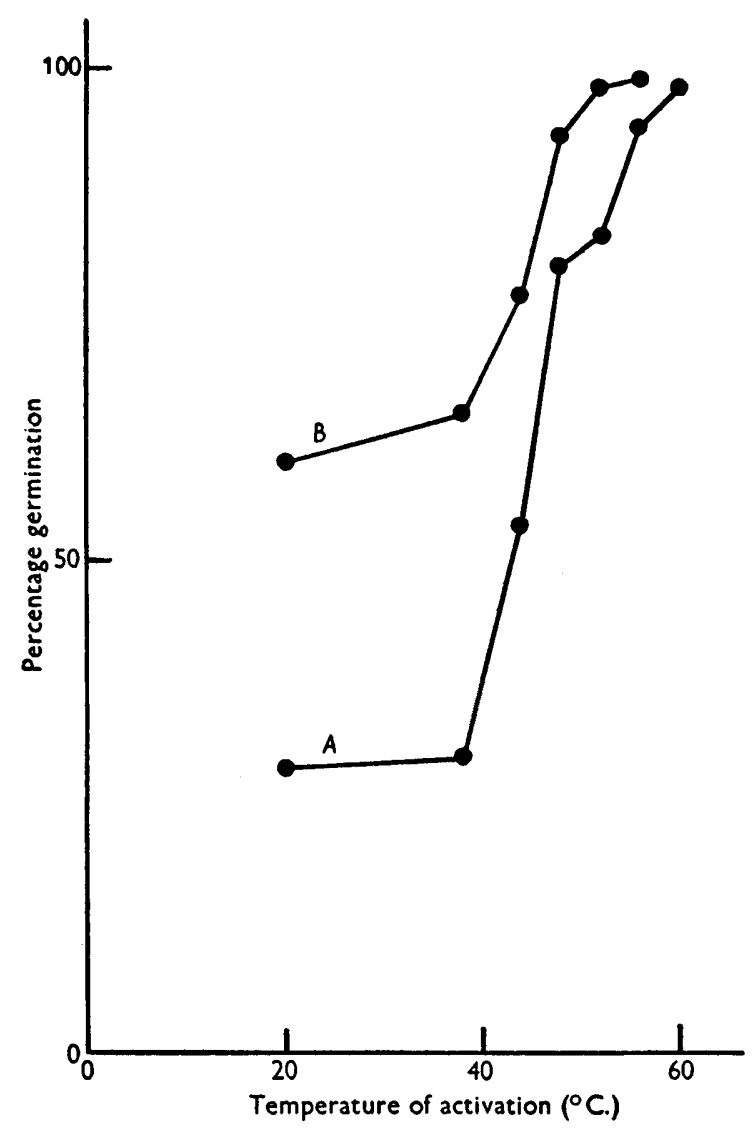

Fig. 3. The effect of $1 \mathrm{hr}$. preheating on germination of $B$. cereus spores in $1 \mathrm{~mm}$-adenosine at $37^{\circ}$. Percentage germination at $\mathrm{A}, 10 \mathrm{~min} . ; \mathrm{B}, 20 \mathrm{~min}$.

(b) to stimulate complete germination in adenosine in $2-5 \mathrm{~min}$. This effect was retained indefinitely.

The rate of germination of preheated spores in $1 \mathrm{~mm}$-adenosine was roughly proportional to the temperature of preheating. The effect of heating a spore suspension which germinated slowly in adenosine for $1 \mathrm{hr}$. at several temperatures between 37 and $60^{\circ}$ is illustrated in Fig. 3. Heating for $1 \mathrm{hr}$. at temperatures below $40^{\circ}$ had relatively little stimulatory effect, although germination became more rapid and complete after several weeks storage of the aqueous 
spore suspension at room temperature or even at $3^{\circ}$. This stimulation appeared not to be due to the liberation of soluble material during heating or storage: heated or stored spores after resuspension in distilled water still germinated rapidly in adenosine alone. The tendency of spores of the laboratory strain of Bacillus cereus to germinate and disintegrate slowly on storage has already been mentioned. There was, however, no tendency to spontaneous germination after heat treatment, although the germination exudate from $B$. megaterium had some stimulatory effect on these heated spores.

The stimulatory effect of preheating depended, as with Bacillus megaterium, on the presence of water. The rate of germination in 1 mM-adenosine was not increased when dried spores were heated for $1 \mathrm{hr}$. at $60^{\circ}$ in the presence of $\mathrm{P}_{2} \mathrm{O}_{5}$. The effect of preheating in the presence of $100 \mathrm{~mm}$ - and $200 \mathrm{~mm}$-sodium fluoride was also determined. In these tests, the spores were centrifuged after heating then resuspended in adenosine solution. Heat activation was completely suppressed by $200 \mathrm{~mm}$ - and partly suppressed by $100 \mathrm{~mm}$-sodium fluoride.

Inosine, guanosine and xanthosine were then tested at $1 \mathrm{~mm}$ as germination stimulants. Inosine was found to be more effective than adenosine: with unheated spore suspensions which germinated slowly and incompletely in adenosine (see above) inosine stimulated complete germination within $15 \mathrm{~min}$. Guanosine and xanthosine were considerably less effective than adenosine. They gave less than $20 \%$ germination in $1 \mathrm{hr}$. when tested alone or in the presence of $1 \mathrm{~mm}$-tyrosine $+1 \mathrm{~mm}$-alanine.

A less complete study was made of the behaviour of Bacillus cereus, NCTC 8035, and of a virulent and an avirulent strain of $B$. anthracis. Unheated spore suspensions of $B$. cereus, NCTC 8035 , germinated less rapidly in TMB and the AdTAl mixture than those of the laboratory strain, e.g. $60 \%$ in $30 \mathrm{~min} ., 90 \%$ in $1 \mathrm{hr}$. The substitution of inosine for adenosine in the AdTAl mixture produced complete germination in $30 \mathrm{~min}$. Inosine tested alone did not, as was the case with the laboratory strain, stimulate rapid and complete germination of these unheated suspensions. In the presence of $1 \mathrm{Mm}$-inosine or $1 \mathrm{Mm}$-adenosine there was less than $20 \%$ germination in $1 \mathrm{hr}$. After heating the aqueous spore suspension for $1 \mathrm{hr}$. at $60^{\circ}$ germination was complete in TMB, AdTAl, InTAl, adenosine and inosine, within 15 min.

Results very similar to those just described for Bacillus cereus, NCTC 8035, were obtained with the virulent and the avirulent strain of $B$. anthracis. Inosine at $1 \mathrm{~mm}$ stimulated rapid and complete germination of preheated but not of unheated suspensions and was consistently more effective than $1 \mathrm{~mm}$ adenosine when tested in the presence of $1 \mathrm{~mm}$-tyrosine $+1 \mathrm{~mm}$-alanine.

\section{Conversion of adenosine to inosine by spores of \\ Bacillus cereus}

Since inosine stimulated rapid germination of unheated spore suspensions of the laboratory strain of Bacillus cereus and was consistently more effective than adenosine for spores of B. cereus, NCTC 8035, and for the virulent and avirulent strains of $B$. anthracis, it seemed possible that inosine might be in 
fact the germination stimulant, and that germination in a given suspension might depend to some extent on its capacity to convert adenosine to inosine. On this basis, the increased rate of germination in adenosine after heat treatment could be ascribed to activation of an adenosine deaminase system. We therefore attempted to assess adenosine deaminase activity in resting spore suspensions disintegrated before and after preheating.

Tests made with intact spores of Bacillus cereus gave results which were difficult to interpret owing to germination occurring during incubation for $30 \mathrm{~min}$. with adenosine. Inosine could not be detected in the medium after paper ionophoresis and examination of the paper in ultraviolet light but two new ultraviolet absorbing spots appeared in positions taken up by adenine and hypoxanthine. These components appeared when germinated spores were incubated with adenosine. It therefore seemed that an extract from disintegrated resting spores would provide a better test system, although disintegration itself might introduce some ambiguity (see below).

Preliminary experiments suggested that disintegrated resting spore suspensions of the laboratory strain of Bacillus cereus converted adenosine to inosine and also partly to adenine and hypoxanthine but that there was no correlation between the rate of germination of a given suspension in adenosine and the deaminase activity of the spore extract. Thus, suspensions which germinated very slowly and incompletely in adenosine gave extracts which had a relatively high deaminase activity as crudely assessed by a visual comparison of ultraviolet absorbing spots. Similarly, although preheating greatly increased the germination rate in adenosine, there appeared to be no marked increase of deaminase activity in the spore extract. It was interesting to find that although the enzyme survived heating in the intact spore, it was completely destroyed in the extract by heating at $60^{\circ}$ for $15 \mathrm{~min}$. Deaminase activity was also found in spore extracts from the avirulent strain of $B$. anthracis but could not be detected in $B$. megaterium spore extracts.

\section{DISCUSSION}

The results obtained with Bacillus megaterium spores suggest the possibility that in this organism, at least three systems are operating to produce spontaneous germination after heat treatment:

(i) A system $\mathbf{H}$ susceptible to heat-activated reversible hydrolysis $\mathrm{H}+\mathrm{H}_{2} \mathrm{O} \rightleftharpoons \mathrm{H}^{\mathrm{w}}$ with a critical temperature in the region of $45^{\circ}$.

(ii) A system G, which controls germination, depending for its activity on the presence of $\mathrm{H}^{\mathrm{w}}$ and functioning within certain limits of temperature not yet closely defined.

(iii) An autocatalytic process, C, due to the stimulatory effect of the germination exudate on heat treated cells.

Thus it might appear that system $G$ does not function at $45^{\circ}$, although after prolonged heating at this temperature $\mathbf{H}^{\mathrm{w}}$ is available. Germination does not occur until the temperature falls, but can still be avoided by rapid cooling to $0^{\circ}$ at which temperature $\mathbf{G}$ is again inactive. 
It was interesting to find that the germination exudate itself stimulated the germination of heat-activated spores. Stewart \& Halvorson (1953) found alanine racemase activity in resting spores of several species of the genus Bacillus, and suggested that the ability of these spores to convert the germination stimulant $\mathrm{L}$-alanine to the inhibitor $\mathrm{D}$-alanine may represent a survival mechanism, i.e. 'a means of ensuring that the supply of viable spores will not all be used up by a single set of favourable circumstances'. An arrangement tending to produce quite the opposite effect appeared to operate in B. megaterium under the conditions we have described.

In Bacillus cereus, and to some extent in B. anthracis, the substitution of inosine for adenosine produced an effect similar to that of heat treatment. It seemed possible, therefore, that an adenosine-deaminase system present in resting spores might be activated by heat treatment, e.g. by a hydrolytic process such as has been suggested for $\boldsymbol{B}$. megaterium. Using a rather crude method of assessment we have so far failed to demonstrate a low deaminase activity in extracts from spores which germinated very slowly and incompletely in adenosine. It may, however, be wrong to draw conclusions about the enzyme activity of intact resting spores from observations on spore extracts. During disintegration, the whole spore structure upon which its special properties depend is destroyed. If, as seems likely, the protoplasm of the resting spore is anhydrous, disintegration would promote hydrolytic processes thus, we suggest, activating the adenosine deaminase system and masking any similar effect due to preheating. The heat stability of the enzyme in the intact resting spore compared with its heat stability in extracts is specially interesting in this connexion and will be more fully investigated.

We wish to thank Mr S. Bailey and Mrs W. Fryer for technical assistance. Acknowledgement is made to the Chief Scientist, Ministry of Supply, for permission to publish this paper.

\section{REFERENCES}

Curran, H. R. \& Evans, F. R. (1945). Heat activation inducing germination in the spores of thermotolerant and thermophilic aerobic bacteria. J. Bact. 49, 335.

Gladstone, G. P. \& Fildes, P. (1940). A simple culture medium for general use without meat extract or peptone. Brit. J. exp. Path. 21, 161.

GODDARD, D. R. (1939). The reversible heat activation of germination in Neurospora. Cold. Spr. Harb. Symp. quant. Biol. 7, 362.

Hills, G. M. (1949). Chemical factors in the germination of spore-bearing aerobes. The effects of amino-acids on the germination of Bacillus anthracis with some observations on the relation of optical form to biological activity. Biochem. $J .45,363$.

Hull, R. (1939). Study of Byssochlamys fulva and control measures in processed fruits. Ann. appl. Biol. 26, 800.

Mickle, H. (1948). 'Tissue disintegrator. J. R. micr. Soc. 68, 10.

Powell, J. F. (1950). Factors affecting the germination of thick suspensions of Bacillus subtilis spores in L-alanine solution. J. gen. Microbiol. 4, 330.

Powell, J. F. (1951). Factors affecting the germination of a strain of Bacillus megatherium. J. gen. Microbiol. 5, 993. 
Powell, J. F. (1953). Isolation of dipicolinic acid (pyridine 2:6-dicarboxylic acid) from spores of Bacillus megatherium. Biochem. J. 54, 210.

Powell, J. F. \& Strange, R. E. (1953). Biochemical changes occurring during the germination of bacterial spores. Biochem. J. 54, 205.

Robinow, C. F. (1951). Observations on the structure of Bacillus spores. J. gen. Microbiol. 5, 439.

Stewart, B. T. \& Halvorson, H. O. (1953). Studies on the spores of aerobic bacteria. I. The occurrence of alanine racemase. J. Bact. 65, 160.

Strange, R. E. \& Powell, J. F. (1954). Hexosamine-containing peptides in spores of Bacillus subtilis, B. megatherium and B. cereus. Biochem. J. 58, 80.

Wade, H. E. \& Morgan, D. M. (1954). The analysis of adenosine triphosphate and adenosine diphosphate preparations by paper ionophoresis. Biochem. J. 56, 41 .

(Received 8 December 1954) 